mt. Der Europarat will mit dem Strafrechtsübereinkommen gegen die Korruption die Mitgliedstaaten, zu denen auch die Schweiz gehört, dazu verpflichten, bestimmte Tatbestände unter Strafe stellen. Der Bundesrat hat einen entsprechenden Gesetzesentwurf in Vernehmlassung gegeben, der noch bestehende Lücken im schweizerischen Korruptionsstrafrecht schliessen soll. Im Bundesgesetz gegen den unlauteren Wettbewerb soll neu die passive Privatbestechung unter Strafe gestellt werden. Ins Strafgesetzbuch soll zudem bei der primären Strafbarkeit des Unternehmens der Tatbestand der aktiven Privatbestechung aufgenommen werden.

Die FMH spricht sich in einer Stellungnahme, die nachfolgend wiedergegeben ist, gegen die Einführung neuer Korruptionstatbestände aus. Solche Tatbestände seien bereits mit der Revision des Strafgesetzbuches im Jahr 2000 eingeführt worden und hätten bisher wenig Wirkung gezeigt. Obwohl im Spannungsfeld zwischen Industrie und Ärzteschaft Korruptionspotential bestehe, habe bisher keine Änderung weitverbreiteter und die Unabhängigkeit der Spitäler und Ärzte in Frage stellender Praktiken festgestellt werden können, weil offenbar der politische Wille dazu fehle. Zudem wäre die Definition der neuen Tatbestände zu wenig klar, was vor allem für Belegärzte grosse Rechtsunsicherheit nach sich ziehen würde.

\title{
Beitritt zum Strafrechtsübereinkommen und zum Zusatzprotokoll des Europarates gegen die Korruption sowie Änderung des Strafgesetzbuches und des Bundesgesetzes gegen den unlauteren Wettbewerb
}

\author{
Vernehmlassung
}

Sehr geehrte Frau Bundesrätin

Der FMH-Zentralvorstand beteiligt sich am Vernehmlassungsverfahren, weil er davon ausgeht, dass die vorliegenden Bestimmungen im Gesundheitswesen mehr Probleme schaffen als lösen würden, und weil er feststellt, dass die 1999 beschlossene erste Ausweitung der Korruptionstatbestände im Gesundheitswesen dort keine erkennbaren Änderungen gebracht hat, wo tatsächlicher Handlungsbedarf offensichtlich erscheine.

Wir beschränken uns auf den Aspekt der Privatkorruption (Art. 4a E-UVG). Bestraft wird (gemäss lit. b) wer «als Arbeitnehmer, Gesellschafter, als Beauftragter oder als andere Hilfsperson eines Dritten im privaten Sektor im Zusammenhang mit seiner dienstlichen oder geschäftlichen Tätigkeit für eine pflichtwidrige oder eine im Ermessen stehende Handlung oder Unterlassung für sich oder einen Dritten einen nicht gebührenden Vorteil fordert, sich versprechen lässt oder annimmt.»

Wir erkennen erhebliches Korruptionspotential im Verhältnis zwischen Industrie und Ärzten und Ärztinnen im Gesundheitswesen. Doch die obenerwähnte neue Antikorruptionsstrafnorm gilt für die öffentlichen Spitäler schon seit Mai 2000, und wir stellen fest, dass dort die objektiv gebotene Praxisänderung schlicht nicht stattgefunden hat. Konkret:

- Es besteht Korruptionspotential bei den Medikamentenrabatten an Spitäler - doch es tut sich nichts: Der Preisüberwacher und die Sanitätsdirektionen haben sich erfolgreich dafür eingesetzt, dass die Industrie Medikamente weiterhin mit hohen Rabatten in die öffentlichen Spitäler liefert (diskutiert wird eine Rabattsumme von 100 bis 150 Millionen jährlich). Die öffentlichen Spitäler haben damit alle Veranlassung, ihre Patienten dank Rabatt auf künstlich verbilligte Medikamente einzustellen. Der Pferdefuss: Die soziale Krankenversicherung muss nach Spitalentlassung oft über lange Jahre den hohen Originalpreis (ohne Rabatt...) übernehmen, weil der praktizierende Arzt den im Spital aufs teure Medikament eingestellten Patienten nur mit Schwierigkeiten und medizinischen Risiken auf billigere Medikamente umstellen kann: alte und/oder verwirrte Patienten können sich nur schlecht daran gewöhnen, ein Medikament zu sich zu nehmen, das anders eingepackt ist und anders aussieht als das im Spital «antrainierte»; und die Einstellung auf 
1 Bemerkenswert die Mitteilung eines Lehrbeauftragten des USZ, sein Lehrauftrag sei nicht gestrichen worden, wohl aber das Salär dafür - mit der Begründung, die Uni müsse sparen und der Lehrbeauftragte habe ja noch andere Einkommensquellen. Persönliche Mitteilung an HP. Kuhn, FMH, Februar 2002.

2 Fletcher RH. Who is responsible for the common good in a competitive market? JAMA 1999; 281(12):1127-8.

3 Finzen A. Wir dankbaren Ärzte. Deutsches Ärzteblatt 2002; 99(12):588-91

4 Kuhn HP. Disclosure helps, but is not a panacea. Schweiz Ärztezeitung 2002;83(25):1318-28.

5 Arzt G. Über Korruption, Moral und den kleinen Unterschied. recht 2001;1:41-50.

6 «Nicht im Hinblick auf die Amtsführung belohnt wird dagegen die Lehrerin, die schwachen Schülern gegen Entgelt Nachhilfeunterricht erteilt, solange die Entlöhnung ein adäquates Verhältnis zur Leistung nicht deutlich übersteigt.» Botschaft BBl 1999, S. 5536.

7 Art. 4a lit b E-UVG.

8 Erläuterungen Seite 28. ein leicht anders wirkendes günstigeres Medikament beinhaltet in der ambulanten Medizin oft Risiken, die bei der Einstellung im Spital besser überwacht werden könnten. Mit anderen Worten: Die Industrie weiss schon, weshalb sie dem Spital Medikamente mit Rabatt liefert - doch niemand interveniert.

- Es besteht Korruptionspotential bei der Fortbildungsfinanzierung - doch es tut sich $\mathrm{zu}$ wenig. Die medizinische Literatur geht davon aus, dass wichtige Indizien für die Beeinflussung von Ärzten durch industriefinanzierte Fortbildung bestehen. Doch die Fortbildungsbudgets der öffentlichen Spitäler gehen auch drei Jahre nach Einführung des revidierten StGB stillschweigend wesentlich von industriefinanzierter Fortbildung aus. So wird der Steuerzahler im Kanton bei den direkten Steuern durch ein künstlich tieferes Spitaldefizit dank Gratisfortbildung geschont [1]. Doch: «there is no free lunch» - Leidtragende ist die Sozialversicherung, wenn die industriefinanzierte Fortbildung unbewusst die Untersuchungs- und Behandlungspläne beeinflusst.

- Forschung und Patienten erleiden Schaden durch die finanzielle Bevorzugung produkteorientierter Forschung an den Spitälern - doch Politik und Nationalfonds geben nicht erkennbar Gegensteuer. Dabei ist die Ausgangslage bekannt: Die Industrie kann nur Forschungsuntersuchungen finanzieren, deren Resultat immaterialgüterrechtlich geschützt ist. Forschung ohne direkten Produktebezug sowie die Erforschung bereits zugelassener Produkte [2] können nur über den Staat selbst finanziert werden, weil diese Erkenntnisse nicht patentierbar sind. Also müsste der Nationalfonds schwergewichtig diejenige medizinische Forschung ermöglichen, die nicht von der Industrie finanziert werden kann, doch eine klare Weichenstellung in dieser Richtung wurde weder von der Politik dem Nationalfonds aufgetragen noch von diesem selbst erkennbar in die Wege geleitet. Resultat: Es wird nicht das erforscht, was medizinisch wichtig wäre, sondern das, was privat finanziert werden kann (siehe etwa [3, 4]).

- Es besteht zudem erhebliche Rechtsunsicherheit, welche Spitäler infolge der in vielen Kantonen stattfindenden sogenannten «Privatisierung öffentlicher Spitäler» noch im strafrechtlichen Sinn als öffentliche Spitäler gelten, für die das im Jahr 2000 revidierte StGB anwendbar ist.
Wir erachten es in dieser Situation als nicht zielführend, neue Antikorruptionstatbestände einzuführen, statt die bestehenden durchzusetzen. Dies um so mehr, als ein Teil der Rechtslehre unseres Erachtens zu Recht die mangelnde Klarheit des Bestechungstatbestands schon für Amtsträger kritisiert hat (vgl. statt vieler [5]). Die Ausdehnung auf die Privatbestechung würde unseres Erachtens im Minimum den Papierkrieg aufblasen, wahrscheinlich aber zusätzlich erhebliche Interpretationsschwierigkeiten für die Strafrechtspraxis schaffen. Wir erwähnen als imaginäres Beispiel den Orthopäden bzw. Kardiologen mit eigener Praxis und Belegarztbetten am Privatspital, der an der Entwicklung von Hüftgelenken bzw. an Medikamentenstudien mitwirkt und später mitverantwortlich für die Beschaffungspolitik des Privatspitals für Prothesen bzw. Medikamente ist. Hier stellen sich mit der vorgeschlagenen Gesetzesbestimmung folgende Fragen:

- Nach welchen Kriterien wird entschieden, ob seine Entschädigung für die Mitarbeit an der Prothesenentwicklung bzw. an der Medikamentenstudie «adäquat» war? [6]

- Der Orthopäde bzw. Kardiologe begründet in der Regel als Belegarzt eine einfache Gesellschaft mit dem Privatspital, in welchem er Belegbetten hat, ist also «Gesellschafter» im Sinne dieses Gesetzesentwurfs. Nach welchen Kriterien würde nun entschieden, ob eine im nachhinein als nicht adäquat angesehene Entschädigung für die Produkteentwicklung im Zusammenhang mit einer Beschaffungsempfehlung ans Privatspital «eine pflichtwidrige oder eine im Ermessen stehende Handlung» [7] war? Wie soll konkret der Strafrichter herausfinden, ob die Beschaffungsempfehlung bei adäquatem Honorar für die Forschungsmitarbeit anders ausgefallen wäre?

- Und nach welchen Kriterien würde schliesslich entschieden, wann bezüglich dieser dem Arzt für die Forschungsmitarbeit in der Arztpraxis bezahlten Entschädigung zwischen Arzt und Spital im Hinblick auf dessen spätere Beschaffungsentscheide ein «vertraglich genehmigter Vorteil» [8] vorliegt - eine Vorteilsgenehmigung, die nota bene «auch implizit» [8] vereinbart werden kann?

\section{Mit freundlichen Grüssen}

\section{FMH}

Präsident FMH

Dr. med. H. H. Brunner

Generalsekretärin FMH

Annamaria Müller Imboden 